\title{
Farmacovigilância: um instrumento necessário
}

\author{
Pharmacovigilance: a necessary tool
}

Helena Lutéscia Coêl ho 1

1 Grupo de Prevenção ao Uso Indevido de Medicamentos (GPUIM), Universidade Federal do Ceará. Rua Fausto Cabral 1188, Fortaleza, CE 60155410, Brasil. lutescia@ufc.br
Abstract This paper discusses the potential benefits of pharmacovigilance in promoting rational drug use and the implementation of drug policies, especially drug regulation, based on the WHO International Drug Monitoring Program. The initial development of pharmacovigilance in Brazil is presented and the need for a National Pharmacovigil ance System is stressed.

Key words Pharmacovigilance; Drug Utilization; Drugs

Resumo O presenteartigo discutea contribuição potencial da farmacovigilância na promoção do uso seguro de medicamentos, tendo como referência o Programa Internacional de Monitorização de Medicamentos da OMS (The WHO International Drug Monitoring Program). É enfatizado o papel da farmacovigilância como instrumento facilitador de uma política de medicamentos, em particular como suporte técni co às ações reguladoras nessa área. São apresentadas evidências do desenvol vimento, ainda que inci piente, da farmacovi gilância no Brasil e da necessidade de potencializá-la, através da criação do Sistema Nacional de Farmacovi gilância.

Palavras-chave Farmacovigilância; Uso de Medicamentos; Medicamentos 
Até fins de 1997, o Programa Internacional de Monitorização de Medicamentos da Organização Mundial da Saúde, TheWHO International Drug Monitoring Programme, contava com 49 países membros (Tabela 1), entre os quais cinco latino-americanos, a saber: Argentina, Chile, Costa Rica, Cuba e Venezuela (WHO, 1997; WHO, s.d.). Participar desse programa significa, principalmente, contribuir para que reações adversas desconhecidas e de ocorrência rara a medicamentos novos ou antigos possam ser identificadas e prevenidas. O acúmulo de notificações espontâneas de suspeitas de reações adversas a medicamentos (RAM) e a sua análise sistemática, em nível local, nacional e internacional, geram hipóteses de causalidade que constituem sinais de alerta, divulgados através de boletim aos centros nacionais e disseminados entre profissionais de saúde de todo o mundo (ten Ham, 1992). Tais informes dão origem a estudos experimentais e/ ou observacionais, que contribuem para a avaliação dos riscos evidenciados pelas notificações espontâneas (Inman, 1993; Lawson, 1997).

O principal objetivo do programa tem sido, desde o seu início, estabelecer e manter um método efetivo de detectar reações adversas não reveladas nos ensaios clínicos (WHO, s.d.).

Em que pesem as limitações decorrentes da subnotificação, da qualidade nem sempre satisfatória das informações e do desconhecimento do real número de pacientes expostos, a notificação espontânea é consi derada o método de melhor relação custo-efetividade, em termos potenciais, na detecção de RAM s desconhecidas e raras (Wiholm et al., 1994). Alguns exemplos de problemas clinicamente importantes, detectados ou confirmados por intermédio desse método observacional, são mostrados na Tabela 2.

O sucesso do programa da OMS em detectar RAM precocemente, isto é, quando o número de casos é ainda pequeno, tem sido reconhecido por muitos estudiosos, bem como a sua eficiência em alimentar os centros nacionais com informações relevantes (Fucic \& Edwards, 1996).

O fato de um país estruturar o seu sistema de farmacovigilância e estar integrado à rede de centros nacionais vinculados ao Programa de Monitorização de Medicamentos da OMS não é, certamente, o bastante para definir que o mesmo desenvolve uma política de medicamentos adequada, do ponto de vista da saúde pública. Na verdade, fazer parte dessa rede pode constituir-se um álibi utilizado por governos no intuito de aparentar seriedade no trato da questão do medicamento, enquanto entre- gam a definição do mercado farmacêutico aos interesses da indústria e do comércio de fármacos. O oposto, no entanto, parece ser verdadeiro, ou seja, o não possuir um sistema nacional de farmacovigilância reflete a pouca seriedade com que um país trata a questão do medicamento no bojo de suas políticas de saúde e o seu despreparo e inoperância para lidar com essa questão. Isso porque se trata de uma atividade pouco custosa e de implementação gradativa (OMS/OPS/ ANMAT, 1997), cujos desdobramentos potencializam, sobremaneira, a eficácia de uma política de medicamentos.

Em primeiro lugar, é importante ressaltar o suporte técnico que um centro ou sistema nacional de farmacovigilância propicia à autoridade reguladora de medicamentos, facilitando o saneamento do mercado farmacêutico e a abordagem de problemas pontuais nessa área. A sua estruturação gera a necessidade de que instituições e serviços, inclusive a indústria farmacêutica, supram o sistema com dados relativos à utilização de medicamentos (comercialização, distribuição, prescrição e uso), e a pacientes (variáveis sócio-econômicas e de morbi-mortalidade), dados estes que constituem os denominadores farmacoepidemiológicos. Tais denominadores são necessários para a avaliação dos riscos relativos à exposição a medicamentos comercializados, procedimento que confere à farmacovigilância o caráter preventivo (Wiholm et al., 1994).

Além disso, a farmacovigilância pode ser um importante instrumento para a promoção de uma mudança cultural que fomente uma percepção mais cuidadosa dos profissionais de saúde e da população em geral, com relação ao uso de medicamentos. O seu exercício também estimula uma maior preocupação com o ensino da Farmacologia Clínica e da Farmacoepidemiologia nos cursos de formação na área de saúde, bem como em programas de educação continuada (ten Ham, 1992; Kimbell, 1993).

Diante de todos os aspectos vantajosos da farmacovigilância, além da sua praticidade, é forçoso reconhecer que o Brasil não tem justificativa aceitável para se ausentar dessa questão. Se olharmos a listagem das nações que fazem parte do Programa de Monitorização de Medicamentos da OMS (Tabela 1), veremos que ali se encontram realidades tão problemáticas como a nossa e algumas até com menor grau de desenvolvimento técnico na área.

Em que pesem as dificuldades e limitações, as iniciativas na área de farmacovigilância no Brasil, nos últimos anos, vêm proliferando e adquirindo consistência (Arrais, 1996; Rosenfeld, 1998). Na área oficial, em maio de 1995, 
uma portaria da Secretaria de Vigilância Sanitária do Ministério da Saúde (MS/ SVS) (Portaria n. 40 de 09/ 05/95) criou uma comissão cuja finalidade seria planejar a estruturação de um Sistema Nacional de Farmacoepidemiologia (Arrais, 1996). Infelizmente, o projeto preparado pela referida comissão não foi colocado em prática antes da substituição do secretário de vigilância sanitária que a criou. Na gestão seguinte, uma outra proposta foi elaborada por um grupo de técnicos a pedido do novo secretário (MS/SVS, 1997), mas, até maio de 1998, nada havia sido concretizado. Enquanto isso, em diferentes locais do País, profissionais de saúde vêm se iniciando na prática da monitorização de RAM, quase sempre mediante projetos de investigação desenvolvidos no contexto hospitalar, como podemos verificar consultando os livros de resumos dos últimos congressos da Sociedade Brasileira deVigilância de Medicamentos (1996, 1997), da Sociedade Brasileira de Farmácia Hospitalar (1996, 1997) e do Primeiro Seminário Brasileiro de Farmacoepidemiologia (GPUIM, 1996). O interesse por essa área pode ser avaliado, também, pela grande afluência aos cursos de farmacovigilância realizados durante os referidos congressos, bem como pela participação de técnicos brasileiros em treinamentos promovidos pela OMS no exterior.

Uma iniciativa de caráter mais amplo está em curso no Estado do Ceará, onde vem sendo implantado um sistema aberto de monitorização de RAM (Sistema de Farmacovigilância do Ceará - Sinface), por meio de um convênio celebrado entre a Universidade Federal do Ceará (representada pelo Grupo de Prevenção ao Uso Indevido de Medicamentos - GPUIM) e a Secretaria de Estado da Saúde. A estratégia da implantação envolve a divulgação do Sinface entre os profissionais de saúde, a ampla distribuição da ficha de notificação e a implantação progressiva da farmacovigilância em unidades hospitalares do SUS. O trabalho nos hospitais é efetuado através das farmácias hospitalares, com a participação de estudantes do curso de Farmácia da Universidade Federal do Ceará. Pretende-se organizar, em cada hospital, uma comissão de farmacovigilância, envolvendo, além de farmacêuticos, médicos e profissionais de enfermagem. As notificações recebidas diretamente ou recolhidas mediante busca ativa são avaliadas e codificadas de acordo com a metodologia recomendada pela OMS (OMS/OPS/ ANMAT, 1997). Tal análise é feita pelo corpo técnico do Ceface (Centro de Farmacovigilância do Ceará), que é a subunidade do GPUIM dedicada à farmacovigilância. As notificações
Tabela 1

Relação de países membros ativos do programa de monitorização de medicamentos da OMS.

\begin{tabular}{lll}
\hline África do Sul & Espanha & O mã \\
Alemanha & Filipinas & Polônia \\
Argentina & Finlândia & Portugal \\
Austrália & França & Reino Unido \\
Áustria & Grécia & Romênia \\
Bélgica & Holanda & Rússia \\
Bulgária & Hungria & Suécia \\
Canadá & Indonésia & Suíça \\
Chile & Irlanda & Tailândia \\
China & Islândia & Tanzânia \\
Cingapura & Israel & Tchecoslováquia \\
Coréia & Itália & Tunísia \\
Costa Rica & Japão & Turquia \\
Croácia & Malásia & USA \\
Cuba & Marrocos & Venezuela \\
Dinamarca & Noruega & \\
Eslováquia & Nova Zelândia & \\
\hline
\end{tabular}

Membros associados: República da China, Egito, Paquistão, Iugoslávia, Chipre, Irã, Sri Lanka e Zimbabwe.

Fonte: WHO, s.d. The Uppsala Monitoring Centre.

Tabela 2

Exemplos de reações adversas importantes, identificadas ou confirmadas com a ajuda de notificações espontâneas.

\begin{tabular}{ll}
\hline Reação adversa & Droga \\
\hline Anemia aplástica & Fenilbutazona \\
Tromboembolismo & Contraceptivos orais \\
Hemorragia gástrica & Antiinflamatórios não esteróides \\
Colite pseudomembranosa & Lincomicina, clindamicina etc \\
Síndrome oculomucocutânea & Practolol \\
Síndrome de Guillain-Barré & Gangliosídeos \\
Toxicidade hepática & Isoniazida \\
Desordens extrapiramidais & Cinarizina, flunarizina \\
Hipersensibilidade aguda & Acetaminofen \\
Fibrose & Ergotamina
\end{tabular}

Fonte: Wiholm et al. (1994); Venning (1983); Capellà \& Laporte (1993).

são de caráter confidencial e o notificador recebe uma carta de agradecimentos, que pode ser acompanhada de informações referentes ao assunto, conforme a necessidade ou solicitação. Após a codificação, as notificações são acumuladas em banco de dados para sua análise sistemática.

De dezembro de 1996 a abril de 1998, o Ceface registrou 95 notificações de RAM e seis 
queixas técnicas relativas a medicamentos. A freqüência de notificações vem aumentando à medida que mais profissionais tomam conhecimento da existência do sistema. $O$ fato de não se aceitarem centros isolados como integrantes da rede da OMS impossibilita a acumulação dos casos registrados pelo Ceface na base de dados do programa internacional, ficando a depender da criação de um centro nacional ou do credenciamento do Ceface pelo Ministério da Saúde. Isso não invalida o trabal ho realizado, tendo em vista o aprendizado e acúmulo de informações que representa, além da possibilidade de detecção de problemas raros ou característicos da nossa realidade.

É claro que a efetividade de um sistema de farmacovigilância depende do grau em que a autoridade responsável pela vigilância de me- dicamentos é capaz de tomar medidas reguladoras relativas a fármacos comercializados (ten Ham, 1992). É evidente, também, que um mercado farmacêutico saturado de produtos questionáveis e de qualidade duvidosa, como o brasileiro, requer muito mais um saneamento do que um acompanhamento (Arrais, 1997). Por outro lado, problemas relativos a deficiências na qualidade da atenção médica, farmacêutica e de enfermagem também contribuem sobremaneira para maus resultados no uso de medicamentos (Bates et al., 1995). Essas constatações podem nos conduzir a pensar que outras prioridades antecedem a estruturação do sistema de farmacovigilância do Brasil. Tal impasse poderá ser resolvido, na prática, pela utilização da farmacovigilância como um canal para o enfrentamento das dificuldades aqui apontadas.

\section{Referências}

ARRAIS, P. S. D., 1996. Farmacovigilância: até que enfim no Brasil. Revista Saúde em Debate, 49/50:8082.

BATES, D. W.; CULLEN, D. J.; PETERSEN, L. A.; SM ALL, S. D.; LAFFEL, G.; SWEITZER, B. J.; SHEA, B. F.; HALLISEY, R.; VANDER, M. V.; NEMESKAL, R. \& LEAPE, L. L., 1995. Incidence of adverse drug events and potential adverse drug events - implication for prevention. JAMA, 274:29-43.

CAPELLÀ, D. \& LAPORTE, J. R., 1993. La notificación espontánea de reacciones adversas a medicamentos. In: Principios deEpidemiología del Medicamento (J. R. Laporte \& G. Tognoni, orgs. ), 2a ed., pp. 147-170, Barcelona: Ediciones Científicas y Técnicas.

FUCIC, H. \& EDWARDS, I. R, 1996. Impact and credibility of the WHO adverse reaction signals. Drug Information Journal, 30:73-80.

GPUIM (Grupo de Prevenção ao Uso Indevido de Medicamentos), 1996. I Seminário Brasileiro de Farmacoepidemi ol ogia. Resumos. Fortaleza: GPUIM.

INMAN, B., 1993. 30 years in postmarketing surveillance. A personal perspective. Pharmacoepidemiology and Drug Safety, 2: 39-258.

KIMBELL, K. H., 1993. Thirty years after thalidomide: still plenty to do. International Journal of Rysk and Safety in Medicine, 4:1-12. 
LAWSON, D. H., 1997. Pharmacovigilance in the 1990s. British Journal of Pharmacology, 44:109110.

MS/ SVS (Ministério da Saúde/ Secretaria de Vigilância Sanitária), 1997. Reunião Técnica para a Estruturação do Sistema Nacional de Vigilância Sanitária. Relatório Final. Brasília: MS/ SVS. (mimeo.)

OMS/OPS/ ANM AT (Organización Mundial de la Salud/Organización Panamericana de la Salud/Administración Nacional de Medicamentos, Alimentos y Tecnología M édica), 1997. Reunión para la Implementación de la Guía para el Establecimiento y Funcionamiento de Centros de Farmacovigilancia en América Latina. Buenos Ayres: Ministério de la Salud de la República Argentina.(mimeo.)

ROZENFELD, S., 1998. Farmacovigilância: elementos para discussão e perspectivas. Cadernos de Saúde Pública, 14:237-264.

SOCIEDADE BRASILEIRA DE FARM ÁCIA HOSPITALAR, 1996. I Congresso Nacional da SociedadeBrasileira deFarmácia Hospitalar. Resumos. São Paulo: Sociedade Brasileira de Farmácia Hospitalar.

SOCIEDADE BRASILEIRA DE FARMÁCIA HOSPITALAR, 1998. II Congresso Nacional da Sociedade Brasileira de Farmácia Hospitalar. Resumos. Belo Horizonte: Sociedade Brasileira de Farmácia Hospitalar.
SOCIEDADE BRASILEIRA DE VIGILÂNCIA DE MEDICAMENTOS (Sobravime), 1995. III Congresso Brasileiro de Vigilância de Medicamentos. Resumos. São Paulo: Sobravime.

SOCIEDADE BRASILEIRA DE VIGILÂNCIA DE MEDICAM ENTOS (Sobravime), 1997. IV Congresso Brasileiro de Vigilância de Medicamentos. Resumos. Curitiba: Sobravime.

TEN HAM, M., 1992. WHO's role in International Drug Monitoring. Post Marketing Surveillance, 5:223-230.

VENNING, G. R., 1983. Identification of adverse reactions to new drugs. I: What have been the important adverse reactions since thalidomide? British Medical Journal, 286:99-202.

WHO (World Health Organization), 1997. National Pharmacovigilance Systems - Country Profiles and Overview. Uppsala: The Uppsala Monitoring Centre.

WHO (World Health Organization), s.d. A Network for Safety. Pursuing the Optimal Balance of Risk to Benefit for Medicinal DrugsWorldwide. Uppsala: The Uppsala Monitoring Centre.

WIHOLM, B. E.; OLSSON, S.; MOORE, N. \&WOOD, S., 1994. Spontaneous reporting systems outside the United States. In: Pharmacoepidemiology (B. L. Strom, ed.), pp. 139-155. Chichester: Strom. 